\title{
The Multi-Application Survivable Tether (MAST) Experiment
}

\author{
Robert Hoyt, Jeffrey Slostad \\ Tethers Unlimited, Inc. \\ 19011 36th Ave W., Suite F, Lynnwood, WA 98036-5752 \\ phone: 425-744-0400ｅmail: hoyt@tethers.com \\ Robert Twiggs \\ Stanford University \\ 469 Lomita Mall, Stanford, CA 94305-4035 \\ phone: 650-723-8651 email: bob.twiggs@Stanford.edu
}

\begin{abstract}
Tethers Unlimited, Inc (TUI) and Stanford University's Space Systems Development Laboratory (SSDL) are collaboratively developing the Multi-Application Survivable Tether (MAST) experiment, which will obtain data on tether performance, survivability, and dynamics. This data is crucial to the development of operational tether systems for propellantless propulsion and deorbit, formation-flying, and momentum-exchange transportation applications. The first objective of the MAST experiment is to obtain detailed on-orbit data on the survivability of space tethers and other gossamer spacecraft structures in the micrometeorite/orbital (M/OD) debris environment. The MAST experiment will deploy three 1-kg CubeSats along a 1-km Hoytether that incorporates both conducting and nonconducting materials. The middle CubeSat will then slowly translate along the tether, inspecting the tether as it moves and returning data on the rate of damage to the tether by M/OD impacts. The second objective of the experiment will be to study the dynamics of tethered formations of spacecraft and rotating tether systems. This data is required to enable the validation of space tether simulation tools such as TetherSim and GTOSS. The third objective of the experiment will be to demonstrate momentum-exchange tether concepts. In this paper we will present results of initial design studies and analyses of MAST system dynamics and performance.
\end{abstract}

\section{Introduction}

Space tethers have the potential to provide missionenabling capabilities for a number of applications, including propellantless propulsion, ${ }^{1}$ formation flying, ${ }^{2}$ and momentum-exchange transportation., ${ }^{3,4,5}$ Currently, a primary impediment to the development of operational tether systems is the paucity of data on the survivability of space tether structures in the micrometeoroid and orbital debris (M/OD), atomic oxygen (AO), and radiation environments in Earth orbit. The available data on the survival of tethers in the space environment is limited to a few inferred data points from the TiPS, SEDS 1\&2, and TSS experiments, which have experienced tether lifetimes ranging from 4 days in the SEDS2 experiment up to 7 years and counting for the TiPS tether. In order to address this issue, Tethers Unlimited, Inc. (TUI) and the Stanford University Space Systems Development Laboratory (SSDL), are working under a NASA Small Business Technology Transfer (STTR) contract to develop the Multi-Application Survivable Tether (MAST) Experiment, which will use the CubeSat platform to obtain on-orbit data on tether survivability within a very low system mass and program cost. In addition, the MAST Experiment will investigate tethered formation dynamics, momentum exchange using a tether, and technologies relevant to orbital debris mitigation. This paper will provide details of the mission objectives, technologies, system design, sched- ule, and current status of the development of the MAST experiment.

\section{Objectives and Program Overview}

The primary objectives of the MAST experiment are to:

1. Obtain in-situ data on the rate at which a space tether structure will degrade in the LEO space environment;

2. Demonstrate a very small, lightweight tether deployment system suitable for nano- and microsatellite applications;

3. Obtain data on the dynamics of tethered satellite formations;

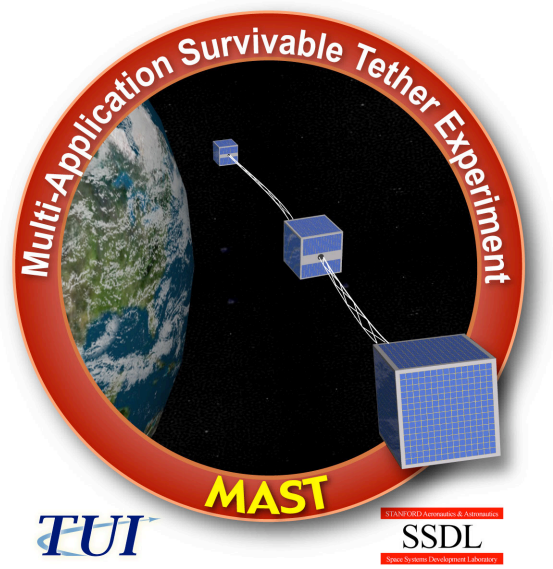

Figure 1. MAST mission patch and experiment concept. 
4. Perform a simple momentum-exchange tether demonstration.

The MAST Mission concept is illustrated in Figure 1. The experiment will fly as a Class- $\mathrm{D}^{6}$ secondary payload, using the Cal-Poly P-POD deployer. ${ }^{7}$ The P-POD will be used to deploy three $10 \mathrm{~cm}$ CubeSats on orbit. These three satellites will be connected by a $1 \mathrm{~km}$ long tether. After deployment, the middle CubeSat will slowly translate along the tether, scanning it for damage due to $\mathrm{M} / \mathrm{OD}$ impacts and $\mathrm{AO}$ degradation, and it will periodically transmit images of the tether to a ground station. Additionally, lightweight GPS and MEMSbased inertial measurement units will obtain data on the dynamics of the three-body tethered formation.

In order to make the development of this mission compatible with both the schedule imposed by the NASA STTR program and the academic schedules of the student members of our team, the development of the MAST experiment has been divided into two stages. In the first, our team is developing a precursor experiment configuration, called the Stanford Tether Release In Neutral Gravity (STRING) Experiment. This experiment configuration will enable testing of the two CubeSats that will be located at the ends of the MAST tether. For the STRING experiment, TUI will supply the tether and tether deployer mechanism, while the SSDL will develop the bulk of the CubeSat systems. The descoped nature of the STRING experiment will enable the SSDL students to complete a flight configuration within their academic program. After a demonstration of these components, TUI and the next generation of SSDL students will then develop the third CubeSat, which will be designed to crawl along the tether and inspect it in the full MAST Mission.

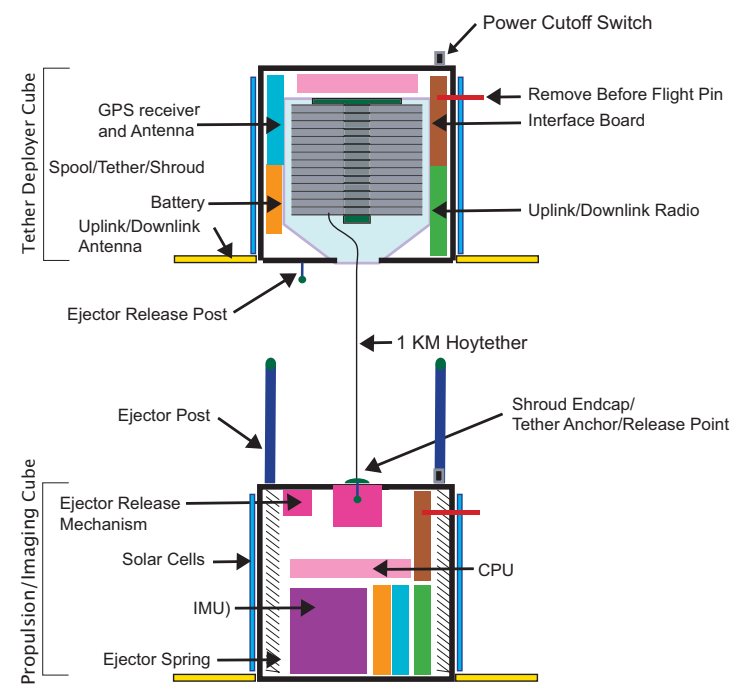

Figure 3 STRING Mission Block Diagram.

\section{Mission Description}

\section{STRING Configuration}

The STRING mission is a precursor developmental experiment intended to demonstrate many of the primary systems and technologies required for the MAST mission. As shown in Figure 3, the STRING mission configuration includes two of the three CubeSats planned for the MAST mission, illustrated in Figure 2. After deployment of the two CubeSats from the P-POD deployer, a timer will trigger a separation mechanism that will push the two nanosatellites apart. The upper CubeSat in Figure 3 will deploy a 1 kilometer long survivable Hoytether in between the two spacecraft. The lower CubeSat has a tether release mechanism that will enable it to separate from the tether upon command. The two STRING CubeSats are nearly identical to the two endbodies in the MAST experiment, with the exception that the Cold Gas and FEAC subsystems will not be implemented in the STRING Propulsion Cube. The primary scientific objective of the STRING experiment is to demonstrate the small tether deployer, the separation mechanism, deployment of a Hoytether in space, and the CubeSat components to reduce technical risk on the MAST mission.

\section{MAST Mission}

As illustrated in Figure 2, the MAST experiment will deploy three 1-kg CubeSats along a 1-km Hoytether that incorporates both conducting and nonconducting materials. The middle CubeSat will contain a crawler system composed of two sets of pinch rollers at either

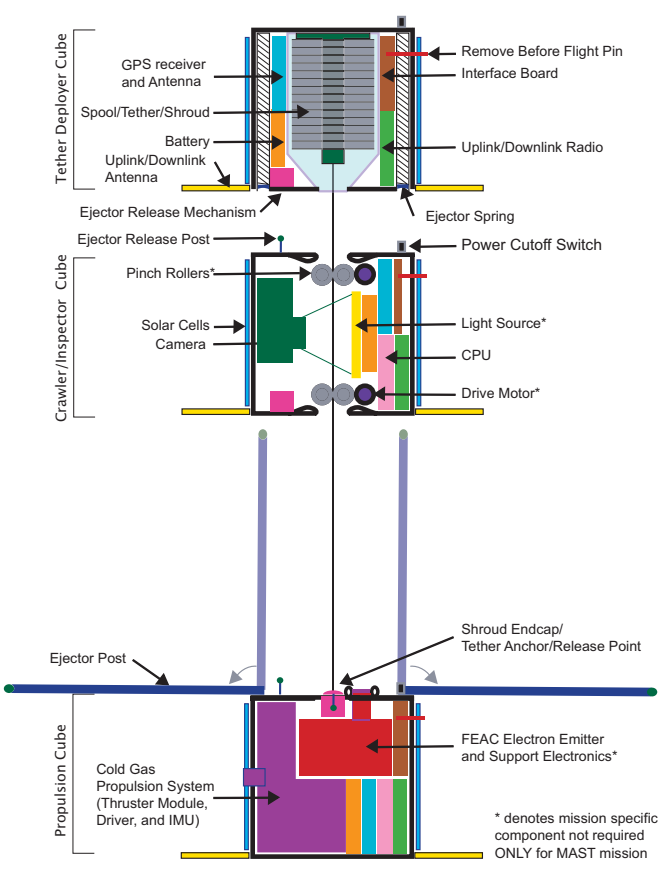

Figure 2. MAST Mission Block Diagram. 
end of the cube as well as an inspection system composed of a backlight and a digital camera.

\section{MAST CONOPS}

The concept of operations (CONOPS) for the MAST Experiment is as follows:

1. The P-POD deployer will first eject the three CubeSats away from the host vehicle. The three CubeSats will initially be held together by separation mechanisms.

2. After a period of time sufficient to ensure that the CubeSats will have drifted a safe distance away from the host vehicle, a timer will activate the separation mechanism between the Deployer and Inspector Cubes. This will allow a set of four balanced springs located inside the four vertical edges of the Deployer Cube to push against the Ejector Posts and eject the Deployer Cube at a velocity of $3 \mathrm{~m} / \mathrm{s}$ relative to the other two Cubes. The initial ejection momentum will be sufficient to pull the tether out of the deployer to its full 1-km length. Passive braking will be used to halt the deployment in a gradual manner to prevent rebound of the Deployer Cube.

3. After deployment of the tether, the Inspector cube will use its pinch rollers to begin moving up the tether. After it clears the Ejector Posts, the posts will swivel away from the tether axis so as to minimize chances of entanglement of the tether on the posts.

4. The Inspector will then slowly inch its way along the tether, taking digital photos of the tether as it goes. It will compress the photos using an appropriate codec and transmit them to the ground station for processing. Our preliminary analyses of communications bandwidth and downlink opportunity frequency indicate that an inspection rate of approximately $10 \mathrm{~m}$ /day should be achievable, enabling the system to scan the entire tether within about three months.

5. After the Inspector has completed its scan of the tether, the Propulsion Cube will then use its ACS systems to null out any significant rotations around the tether axis. The Propulsion Cube will then activate its cold gas thruster to apply a $25 \mathrm{mN}$ impulse for 100 seconds. During this impulse, the attitude sensing and control systems in the Propulsion Cube will hold the slew rate of the cube around the tether's axis to less than 0.1 degree/sec; this rate is commensurate with the capabilities of low-cost MEMS inertial measurement technologies and will suffice to ensure that enough of the impulse is aligned along one general direction. This thrust impulse will then be sufficient to set the tethered system in rotation, as illustrate in Figure 4.

6. The tethered CubeSat system will then be allowed to continue rotating for a period of several weeks to
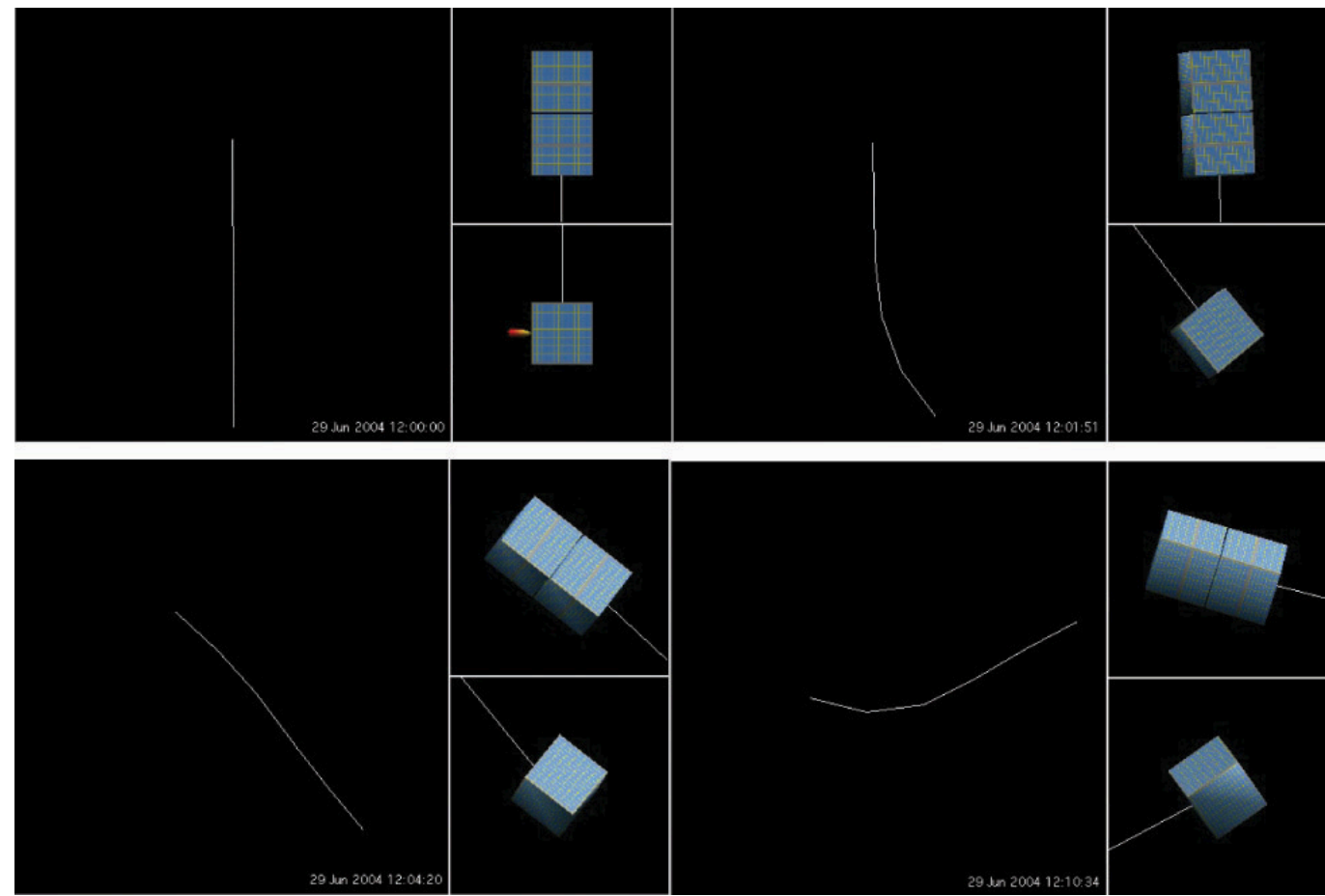

Figure 4. TetherSim ${ }^{\mathrm{TM}}$ simulation of spin-up of the MAST Experiment using a $100 \mathrm{sec}$., $25 \mathrm{mN}$ impulse from a coldgas thruster, with cubes rolling at $0.1 \mathrm{deg} / \mathrm{s}$ 
several months in order to obtain detailed data on the dynamics of a rotating tethered system.

7. To conclude the mission, the tether release mechanism on the Propulsion Cube will be activated when the Propulsion Cube is at the top of its swing, separating the Propulsion Cube from the tethered system and thereby performing a simple demonstration of tethered Momentum-Exchange. The momentum exchange will toss the Propulsion Cube into a higher orbit while dropping the other two cubes into a deorbit trajectory.

The primary goal of the experiment will be to acquire detailed on-orbit data on the survivability of space tethers in the micrometeorite/orbital (M/OD) debris environment. This data will be obtained by groundprocessing of the images of the tether that the Inspector Cube will transmit down once per day. The mission will also demonstrate electrodynamic tether propulsion using a Field Emission Array Cathode electron emitter. This use will likely be the first on-orbit demonstration of the FEAC technology. Another goal of the mission will be to obtain detailed data on the dynamics of tethered formations of spacecraft and rotating tether systems. Small GPS units onboard each of the Cubes will provide measurements of the dynamic behavior of the tethered system to enable validation of space tether simulation tools such as TetherSim and GTOSS. The final goal of the mission will be to demonstrate momentum-exchange tether concepts.

\section{Mission Philosophy}

The MAST Mission is a REAL Class D mission to demonstrate and evaluate some of the basic elements of tether propulsion. This means that we will attempt to show the benefits of tether propulsions while minimizing cost. We will accomplish this by performing only documentation that is required to guide and coordinate the development and satisfy the launch vehicle requirements. As well, risk will be managed as a resource along with mass, schedule, power, volume, and cost. When applicable, non-space rated components will be used to minimize costs, although sufficient testing will be performed to screen out parts that have manufacturing defects or are not capable of performing in the expected environment. Reliability will be attained by testing, use of flight proven hardware and design, and by cooperative subsystems (such as using the GPS tracking system to provide tether lifetime information along with the inspector cube). In general, the design will be single string, in the sense that there will not be redundant hardware, though every attempt will be made to design the system to allow for a graceful degradation so that the failure of one component or subsystem will not impact others whenever possible.

\section{Subsystem Development}

Significant progress has been made on the development of the systems shared by the STRING and MAST missions, in no small part due to the efforts of the SSDL team. Preliminary configurations of the two cubes in the STRING mission (TED and RALPH) are shown in Figure 5. An overview of some of the subsystems of note follows.

\section{Communications}

The downlink requirements are driven primarily driven by the GPS data and some basic housekeeping data. Based on a preliminary, very conservative analysis, the downlink will need to be capable of up to $1.5 \mathrm{Mbits}$ per day, including overhead for packetization and error correction. This estimate also includes the ability for high speed GPS and IMU data modes, which will only be performed when the downlink capabilities are able to handle the increased load. In addition, the housekeeping data will have variable rates to maximize science data capabilities. Using the existing ground station capabilities at Stanford, initial analysis suggests that during a single pass each day the downlink capability will range from 9.4 to 14.5 Mbits for orbits between 700 and $400 \mathrm{~km}$ respectively. The availability of additional ground stations would greatly increase this capa-
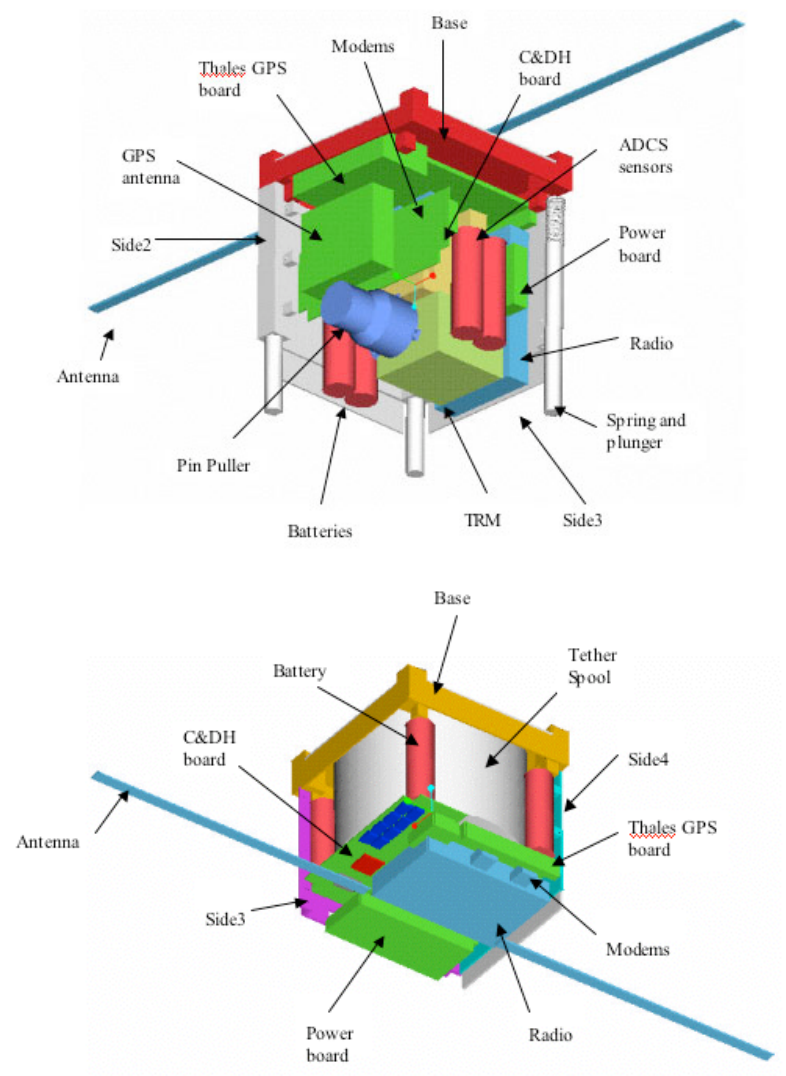

Figure 5. Layout of the Deployer CubeSat (bottom) and the Propulsion CubeSat (top) 
bility, allowing more aggressive science objectives.

\section{Command and Data Handling}

The choice of processors was driven primarily by the processing requirements, the power usage, and the need to provide adequate interfaces to support both the STRING and MAST missions. The MicroChip PIC 18F8720 processor was chosen, giving 128 Kbytes of program memory, a 16 channel 10-bit A/D, 2 RS-232 UART channels, and two $\mathrm{I}^{2} \mathrm{C}$ interfaces. The maximum power usage at 5 Volts is $0.1 \mathrm{~W}$, with a voltage input capability of 4.2 to 5.5 Volts, all in a package massing less than 1 gram. This processor is mounted to the main C\&DH board along with additional memory, and a watchdog timer. Accommodations for a daughterboard to provide additional capabilities for the MAST mission has also been designed onto the board, although a daughterboard will not be utilized for the STRING mission. Single event upsets and latchup will be handled by a watch dog timer.

\section{GPS Unit}

One of the most challenging aspects of the MAST mission design has been the selection of a GPS unit. A GPS unit is required on each of the CubeSats to verify the performance of the tether deployer and obtain data on tethered formation dynamics. The capability of removing the velocity and altitude limits is found primarily in units far too large or power hungry for CubeSat applications. Furthermore, most manufacturers have little interest in removing the limits even if the hardware is capable of performing the necessary phase corrections. The leading contender at the time of the papers submission is the Surrey Satellite Technology LTD SGR-05, shown in Figure 6, which is specifically designed for microsatellite applications.

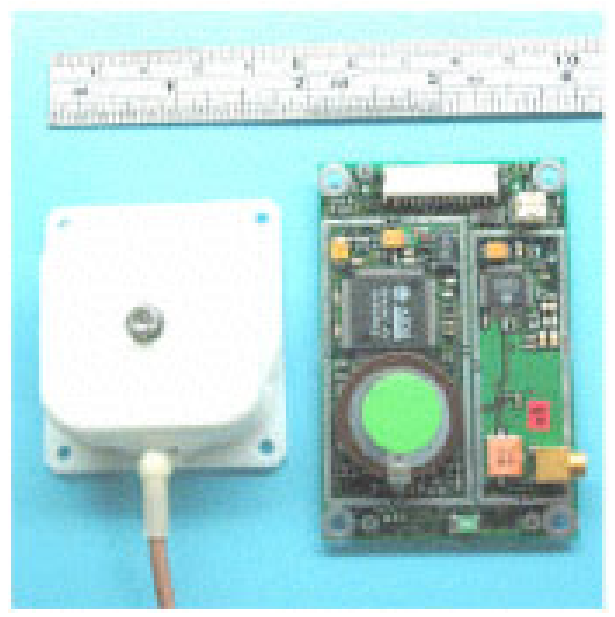

Figure 6. The SST SGR-05 GPS unit.

\section{Power}

The power subsystem consists of one 3.6V Lithium Ion cell per cube, a 5 volt regulator, a capacitor for high current transient loads, and diode protected solar cells. The solar cells will provide $6.15 \mathrm{~V}$ at a nominal 2.2 watts. Due to the high power requirements for the cube and tether release mechanisms, a 1F "Aerogel" capacitor is being investigated for use, although it may require special packaging for vacuum applications.

\section{Tether Deployer}

In order to provide a small, lightweight, and low-cost tether deployment system that will be useful for nanoand micro-satellite applications such as tethered formation flying, electrodynamic drag deorbit, and gravitygradient stabilization, TUI is developing a smaller version of its $\mu$ PET tether deployer for use in the MAST experiment. $^{8}$ A deployment test of the $\mu$ PET deployer is shown in Figure 7. A challenge in designing the deployment system is selecting the right ejection velocity to ensure that the tether deploys at or near its full length without hitting the end of the tether with an excessive velocity that would cause significant rebound. Using the TetherSim ${ }^{\mathrm{TM}}$ code, we have simulated the deployment of the MAST system with a variety of deployment drag profiles based upon past measurements of tether deployment drag. A visualization of the results of one of these simulations is shown in Figure 8. Based upon these simulations, we have identified $3 \mathrm{~m} / \mathrm{s}$ as the optimum ejection velocity.

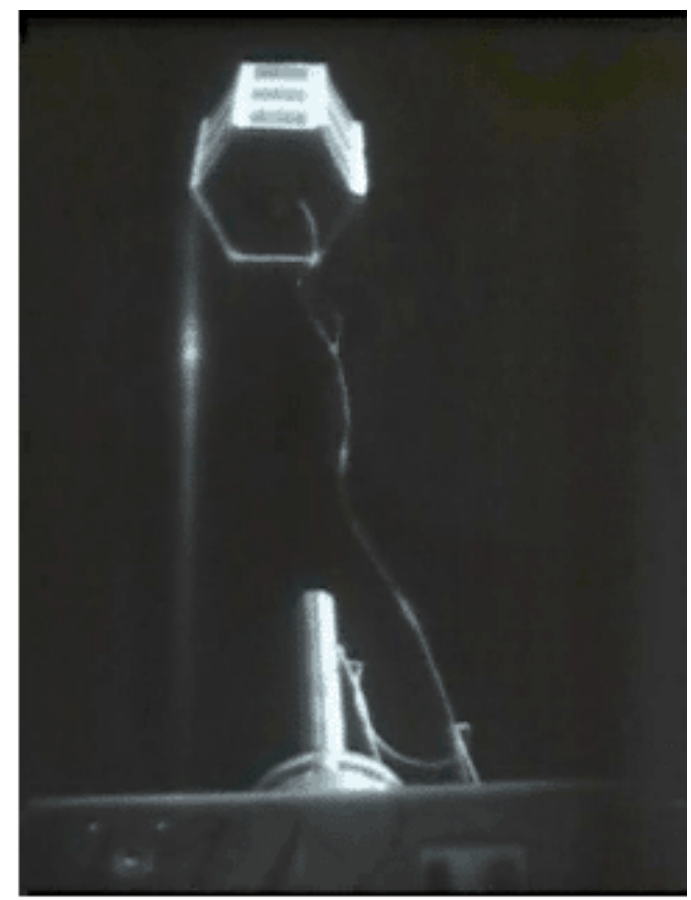

Figure 7. Deployment test of the $\mu$ PET deployer. 

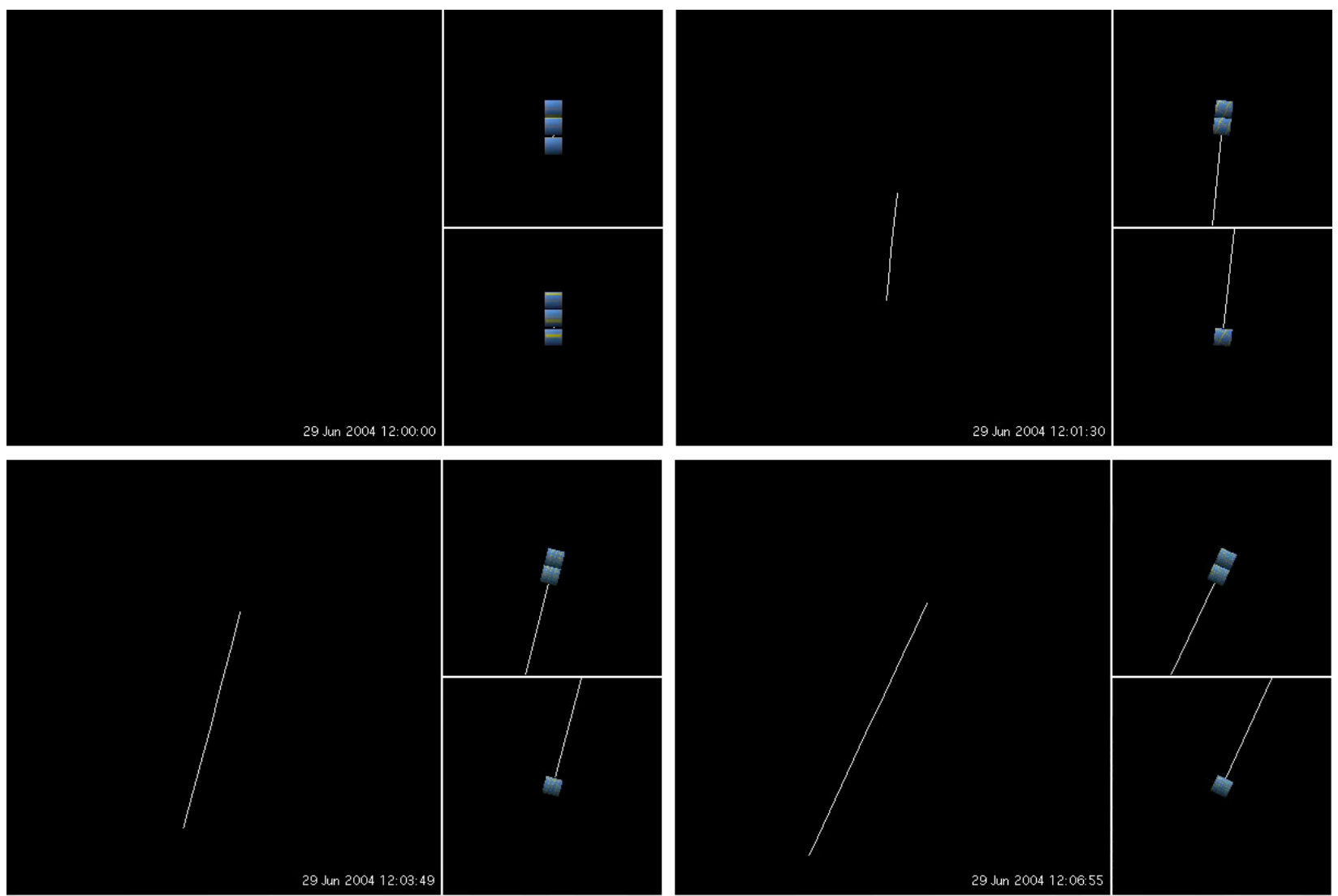

Figure 8. TetherSim ${ }^{\mathrm{TM}}$ simulation of deployment of the MAST system.

\section{Conclusions}

In the six months since the initiation of the MAST STTR effort, we have made excellent progress in refining the mission goals, defining the system architecture, and designing the CubeSat subsystems. We have focused the first year's efforts on developing two of the three CubeSats, the Propulsion Cube and Deployer Cube, in order to enable the SSDL student team to complete the development of these systems within the timeframe of their academic program. These two CubeSats will validate the performance of TUI's nanosatellite-applicable tether deployer, and validate many of the key systems for the MAST experiment. The MAST experiment is anticipated to occur under the Phase II of the NASA STTR program, and will provide invaluable data on tether survivability, tethered formation dynamics, and tethered momentum exchange propulsion.

\section{Acknowledgements}

This research has been conducted under NASA/MSFC STTR Contract NAS8-03037, Kirk Sorenson COTR. The authors wish to acknowledge the hard work and invaluable contributions of the SSDL student team, including Paul Forquera, Tom Taylor, Steve Davis, Ylan Segal, Marty Lichtman, Bulent Altan, Nick Harrison, David Ngo, Neel Vora, and Andy Carmain, as well as advisors David Klinger and BJ Jaroux. 


\section{References}

1. Forward, R.L., Hoyt, R.P., "Space Tethers," Scientific American, pp. 86-87, February 1999.

2. Farley, R.E., Quinn, D.A., "Tethered Formation Configurations: Meeting the Scientific Objectives of Large Aperture and Interferometric Science," AIAA Paper 2001-4770, AIAA Space 2001 - Conference and Exposition, Albuquerque, NM, Aug. 28-30, 2001.

3. Hoyt, R.P., Uphoff, C.W., "Cislunar Tether Transport System," Journal of Spacecraft, Vol. 37, No. 2, pp. 177-186, March-April 2000.

4. Hoyt, R.P., "Design and Simulation of a Tether Boost Facility for LEO->GTO Payload Transport", AIAA Paper 2000-3866, 36th Joint Propulsion Conference, July 2000.

5. Sorensen, K., 2001, "Conceptual Design and Analysis of an MXER Tether Boost Station," AIAA Paper 2001-3915.

6. DoD-Handbook-343, Design, Construction, and Testing Requirements for One of a Kind Space Equipment.

7. Nason, I, Creedon, M, Johansen, N, "CUBESAT PPod Deployer Requirements," May 2002.

8. Hoyt, R.P., et al., "The Proposed RETRIEVE Microsatellite Deorbit Experiment," AIAA Paper 2002$3893,38^{\text {th }}$ Joint Propulsion Conference, Indianapolis, IN, July 17-19, 2002. 